\title{
Bodies need yoga? No plastic surgery! Naturalistic versus instrumental bodies among professions in the Danish healthcare field
}

\author{
Kristian Larsen ${ }^{1,2,3}$ (D) Anette Lykke Hindhede ${ }^{4}$. Mikkel Haderup Larsen ${ }^{4,5}$. \\ Mathias Holst Nicolaisen ${ }^{4,6}$. Frederik Møller Henriksen ${ }^{7}$
}

Accepted: 14 October 2020 / Published online: 9 November 2020

(c) The Author(s) 2020

\begin{abstract}
What characterises the similarities and differences in body investments among professions in the Danish healthcare field? This can be important when healthcare professionals relate to each other and to bodies of patients regarding differences in class, gender, age, and disease group. The study takes inspiration from Bourdieu's sociology and the concept of health capital. We ask whether health capital can explain distinctions in the empirical data? We used the explorative-descriptive method multicorrespondence analysis (MCA) on data from 440 respondents gathered through an online survey. The respondents range from chief physicians to healthcare students. MCA makes it possible to condense frequency statistics and examine patterns of body investments through an examination of variables such as food intake, exercise, and medicine use that are then further related to supplementary variables such as social class and age. The analysis supports the concept of health capital and shows that some groups in the Danish healthcare field have a more instrumental body perception, such as the young age group (e.g. open to surgery, consume medicine, high exercise), while the upper-middle-class and older respondents have a more naturalistic perception of the body (e.g. read often, eat vegetables, practice yoga).
\end{abstract}

Keywords Bourdieu · Multicorrespondence analysis (MCA) · Body investment . Healthcare field $\cdot$ Health capital $\cdot$ Distinction

\section{Introduction}

In the article, we examine bodily health investments among a convenience sample of professionals and students from the Danish health sector. Health ideals and health capital are not fixed. In the 1950s and 1960s, ideals were related to large

Kristian Larsen

kristian.larsen@regionh.dk; krila@oslomet.no

Extended author information available on the last page of the article 
bodies, but today large bodies are stigmatised as representing a loss of self-control (Brewis et al. 2011). Over the past 40 to 50 years, radical changes in the social space have occurred. New forms of classificatory struggles (Tyler 2015) and definitions of values (Skeggs 2011, p. 502) related to body cultures are relevant to study and integrate.

The focal point of our analysis is the concept of embodied health capital, which we claim can explain how individuals perceive and invest in their bodies differently. Health capital is attained through health investment, understood as individual strategies or techniques related to emerging academic discussions on healthism, social equality, and power struggles in professional fields. It is a precondition of health capital, that possessions can be measured. As expressed by Lupton (2016) measuring the body and its performances related to health is not a new phenomenon. Throughout history, the monitoring or the self-tracking of the body has had its different devices and technologies that made possible measuring and thereby also 'selfexperimentation' and self-optimisation (Lupton 2016, p. 32).

The article highlights how physical, mental, and social dimensions of bodily appearances are tied together in modes of health investments, thereby contributing to abovementioned themes in health studies. The concept of Health capital has been theoretically developed as a resource available to young people in Denmark (Larsen and Morrow 2009) and in a Norwegian study of inequality in health (Larsen and Hansen 2014); however, our intention is to further examine health capital among professions in the Danish healthcare field by using multicorrespondence analysis (MCA). MCA is an exploratory data analysis technique that can identify groups of individuals with similar response patterns on survey items and plot associations between categories. It has previously been applied to analyses of a wide range of phenomena from drinking patterns in work-life contexts (Järvinen et al. 2014) to cultural consumption and politicomoral stances as markers of class position (SkjøttLarsen 2012). Although health capital relates to economic, cultural, and social capital via money, education, and social networks, we claim that health capital provides a unique insight into the various ways people invest in their body. There is a growing emphasis on health and health promotion in Nordic work-life (Larsen and Harsløf 2019), and we expect this is particularly actualised in the healthcare field.

We have typified health capital with five distinctive modes of body investment: the surgical body (surgical and cosmetic interventions), the chemical body (biochemical and chemical tools), the nutritional body (eating habits and practices), the physical body (physical exercise), and the mental body (stimulation of the mind). We support the notion that health capital has potential as a new form of capital that compensates or subsidises economic, cultural, and social capitals and can be deployed in diverse positions in different fields and the social space, including in work-life contexts. While some research has already been carried out, it remains only suggestive (see Larsen, Cutchin and Harsløf 2013; Larsen 2009). Hence, this article explores differences and similarities in body investments among professionals and students from the Danish health sector and discusses how embodied health capital can provide an explanatory framework for these distinctions. 


\section{Theoretical background: embodied health capital}

The development of health capital is rooted in the sociology of Pierre Bourdieu and especially the concepts of social space, social field, distinction, habitus, and forms of capital (Bourdieu 1977, 1984, 1986), and it is inspired by the historical epistemology (Bachelard 1968; Canguilhem 1988) advocating for empirical-theoretical research that calls for new understandings and a 'break epistemology' as part of the craft of sociology (Bourdieu et al. 1991). With its 'tyranny of the Market' (Bourdieu 1998, 2001), neoliberalism has transformed the state (Bourdieu and Farage 1994; Bourdieu 1999), implying the dominance of the right hand of the state (technocratic state) and the withdrawal of the state in policy areas such as social housing, public service broadcasting, and hospitals formerly under state jurisdiction, which consequently have enhanced processes of individualisation (Bourdieu 1998).

From a historical perspective, a number of phenomena together constitute a 'taste for health'. Bourdieu (1985) speaks of an immersion in a wider field of symbolic manipulation. Over time, science and, in particular, health science have supplanted religious symbols in the social space, installing psychologists, psychoanalysts, doctors, sexologists, life coaches, body therapists, natural healers/therapists, and instructors as the 'new priesthood' (Bourdieu 1981). These positions take part in the struggle to provide the 'laity' with advice about how to live via health, healing, and spiritual and bodily care. Another feature affecting the taste for health relates to how the ingestion of food and beverages over time, established as relatively autonomous fields of knowledge (e.g. 'eating'), becomes 'nutrition'. An additional feature relates to how ritual games and festive entertainment-again from a historical perspective-are transformed into 'sport' and, along the way, established as a competitive field (Bourdieu 1981). The concept is also inspired by other scholars in the field of sociology of health and illness, especially those expanding on Foucault. The works of Nikolas Rose $(2003,2007)$ has parallels in terms of its description and analysis, compared to the concept of health capital. As Rose (2003, p. 58) says: 'where Foucault analysed biopolitics, we now must analyse bio-economics and bioethics, for human capital is now to be understood in a rather literal sense-in terms of the new linkages between the politics, economics and ethics of life itself'. Here, Rose (2003) is interested in the general transformation of the politics, economics, and ethics of life itself, not-like Bourdieu-in differences between bodies and the fact that they are asymmetrically valued.

Bourdieu (1977) described three mutually dependent dimensions of cultural capital: the embodied, objectified, and institutionalised. Inspired by this, we have conceptualised health capital in a similar way based on (Larsen et al. 2013); however, this article solely focuses on health capital in its embodied state. We construe health capital through five different modes of body investment. Health capital as a sociological concept is concerned with social advantage, and these different and opposed investment styles are practised more often as everyday activities than thought of as related to health in a medical sense. In the following, we present the five types of body investment and explain how they are measured. 
The surgical body refers to investments in surgical interventions with the purpose of optimising the social impact of bodies. Examples of the surgical body are investments via technologies and clinics that specialise in implants, facelifts, obesity surgery, or in designing (i.e. enlarging/reducing or correcting faces, noses, buttocks, breasts, or genitals). Two items in the survey tap into the surgical body, asking about the subject's opinion on body alterations and cosmetic surgery.

The chemical body is a form of investment in or on the body via a broad range of biochemical and chemical tools, medicine, and pharmaceutical products and technology. Examples are use of medical, smart or illegal drugs, various health and skincare products to enhance cognitive, mental or physical capacities. One item measures the chemical body; a question on use of drugs against physical illness or pain.

The diet body refers to ways of correcting and optimising the body through diet and eating habits. We measure this by an index based on different food categories (i.e. nuts, salads, and organic foods) and a question about the intake of supplements, vitamins, and minerals.

The physical body is a form of investment through work on the 'outer body'. Examples are investments through fitness programmes carried out at home or at a fitness centre using apps or coaches or through sports and other types of exercise. Three items tap into this by asking about frequency of exercise (in hours), the body mass index of the subject (BMI), and whether the respondent prefers a holiday revolved around physical activities.

The mental body is a body investment strategy acquired through time and energy spent on the 'inner body', on literature, science or art, or on performing yoga or mindfulness. We made two binary indicators assessing if the subject carries out wellness-related activities (e.g. massage or body wrap treatments) and new age activities (e.g. yoga or tai chi), respectively. We also include an item on reading frequency tying the mental body to embodied cultural capital (Bourdieu 1986).

Overall, we examined whether the five types of body investment could be reconstructed empirically, thereby illuminating certain patterns of body investment among the participating subjects. We apply 12 variables measuring modes of body investment as well as three demographic items, social class, age, and gender, to enrich the interpretation of the map.

The application of health capital as the theoretical framework would at first hand seem like a theory-testing endeavour, something that does not correspond to the explorative nature of MCA. However, our intention to trace certain body investment trends among employees and students in the healthcare field allows for new explanations to come to light, which would then call for further theorising (Bertilsson 2004). The purpose is thus to characterise body and health investments as phenomenon without application of any strong a priori assumptions. Nonetheless, it is necessary with theoretical insights to be able to conduct interpretative research categorisations. This is following the principles in sociological research involving epistemological break, construction, and applied rationalism, but also integrating the relational thinking (Bourdieu et al. 1991). Therefore, the survey questionnaire is to a certain extent theory-informed and built around measures of the five types of body investment, but it is not theory-determined (Carleheden 2016). Hypothetically, they could be incoherent, and no obvious pattern would appear in the clouds (for definition of 
cloud, see 'Data and methods'). In this respect, empirical social science is in a constant state of flux, since it is always approximating the social reality studied, which is dynamic in nature (Bourdieu et al. 1991). In this respect, health practices and the development of health capital as a concept are moving targets, where new information, technologies, and advice are constantly revised and presented. This applies to questions of what is healthy/unhealthy, new modes of body investment, and so forth.

Before the analysis, we present a data and methods section. First, we provide a brief description of MCA and why it is a relevant statistical technique for this type of research. After, we discuss the design of our survey questionnaire and explain the sampling procedure.

\section{Methodological background and data: multiple correspondence analysis}

To discover specific body investment trends among medical professionals and students, we use multicorrespondence analysis. MCA is a geometric data analysis method that translates a covariance matrix into coordinate points in a geometric space (Benzecri 1969). By way of visualisation, the complex connections between multiple outcomes appear more comprehensible and thus make interpretation more intuitive. In the methodological literature, it is described as a variant of principal component analysis and factor analysis, when the analysed variables are categorical (Abdi and Valentin 2007). MCA provides us with two visual outputs: (1) the cloud of categories (see Fig. 2 in 'Analysis'), where the points represent variable categories, and (2) the cloud of individuals (see Fig. 4 in 'Analysis'), where the points represent individuals. The set of all distances between individuals defines the cloud of individuals, where a greater distance between two individuals indicates dissimilarities in response patterns. Individuals choosing infrequent categories are placed in the outskirts of the map (Roux and Rouanet 2010). The same logic applies to the cloud of categories following the same methodological characteristics as the cloud of individuals vis-à-vis dimensionality and variance. Here, categories positioned in proximity indicate agreement between individuals on those parameters. Infrequently chosen categories are positioned further away from the centre.

The MCA procedure goes hand in hand with a Bourdieusian approach attempting to illuminate patterns of investment and preferences (Bourdieu 1984). Bourdieu himself viewed the method as a relational procedure that expresses his view of how multidimensional fields constitute social reality (Roux and Rouanet 2010). Furthermore, Järvinen et al. (2014) emphasise how the method is convenient in identifying latent variables mapping them as dimensions wherein the variables are located. Di Franco (2016) explains that MCA allows for a flexible research approach, as it does not assume an a priori relationship between the categorical variables under investigation and their distribution. For these reasons, it is usually applied as an explorative tool to examine the patterns across data. However, two principles for improving the results are widely applied; recoding of variables to balance cases within the categories and balancing the number of categories per single variable (Di Franco 2016; Roux and Rouanet 2010). Table 1 shows our best effort to apply to these principles 


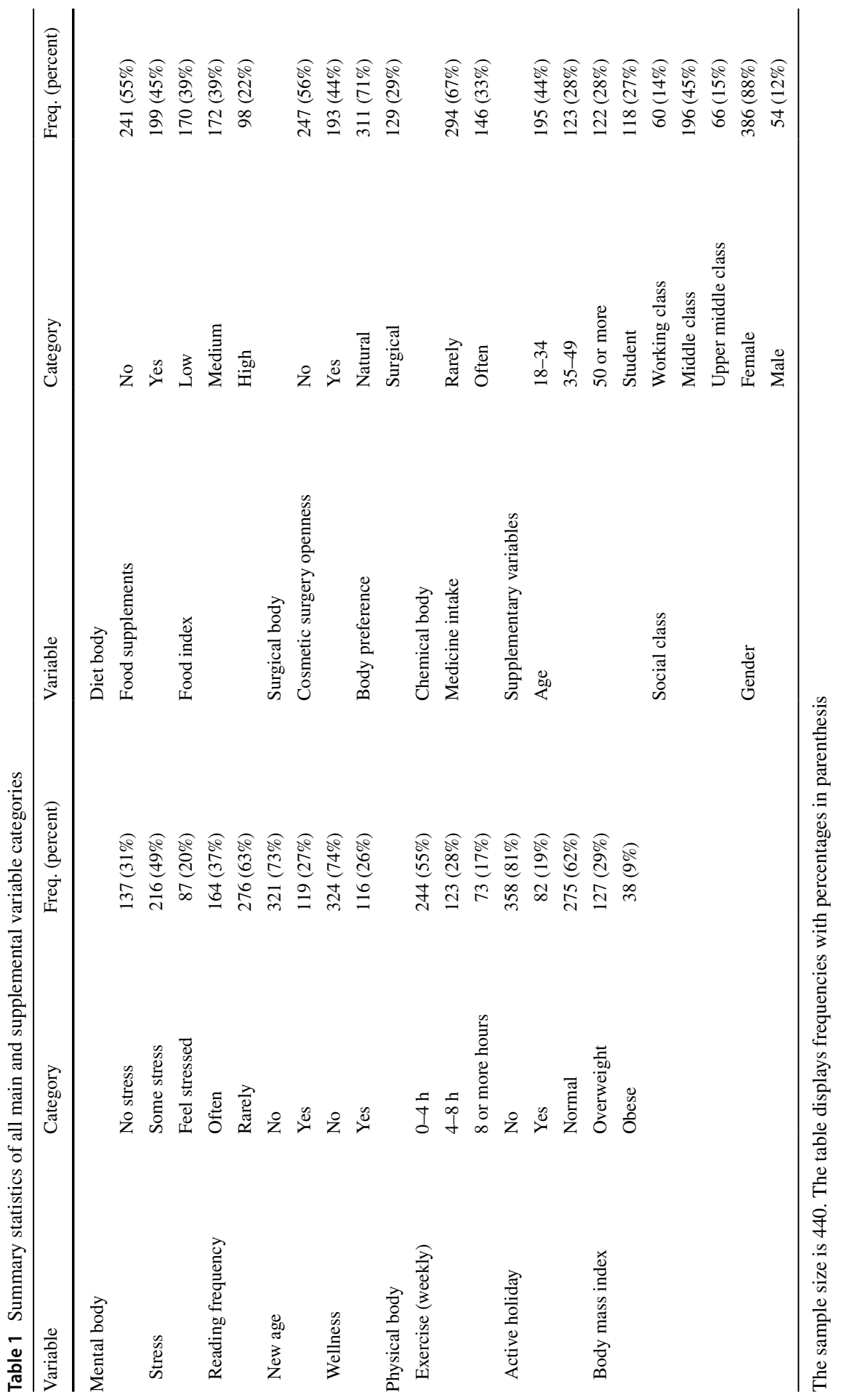

尔 
and still retain meaningful categories for interpretation, apt at studying phenomena that are structured by the interaction of many different variables (Table 2).

\section{Survey design and sampling procedure}

In the analysis, we utilise a convenience sample of 440 participants (full response) collected in the period from medio 2017 to ultimo 2018. There is no convention about a minimum case requirement; however, too few cases create unreliable clouds. Di Franco (2016) proposes a lower threshold of 20 cases per active category which our sample contains (see 'Table 1'). Survey participants were successively recruited through colleagues and friends employed in the primary and secondary sector, public and private institutions, and higher education institutions. The first author sent introductory information about the research project by e-mail, which contained a link to the survey. Applying chain-referral sampling or snowballing, participants then recruited further subjects among colleagues or students. The questionnaire was composed of 12 sections and took approximately 25-30 min to answer. As we are exploring a new concept, it was not feasible to draw on more standardised survey questions. Rather, the questionnaire is inspired by the first author's previous research about inequality, the body, and health (Larsen and Morrow 2009; Larsen et al. 2013). The survey is designed based on the expectation that bodily performances and activities are valued and invested differently. Like previous Bourdieusian studies, the survey questions reflect self-evaluated praxis, taste, and preferences which in turn illuminate similarities and dissimilarities in the sample. Demographic covariates are used as supplementary elements to enrich the interpretation of the data and guide the analysis. This relates to how habitus on a bodily and unconscious level 'reads the social', instead of it being solely explicit for the individual (Bourdieu 1977).

The sample is composed of different types of professionals and students from the Danish healthcare sector (see 'Table 1'). Among the respondents, about $27 \%$ are studying to become healthcare assistants, nurses, or doctors, which we group together as students. Around $45 \%$ have a medium income level and a bachelor's degree, medium, or long cycle higher education and consequently coded as middle class. Approximately $15 \%$ have a high income ( $>100,000$ euro annually) and are coded as upper-middle class. The remaining $14 \%$ have completed a vocational programme, short-cycle higher education or similar and are coded as working class. The sample has a large gender

Table 2 Eigenvalues, variance, and adjusted variance exhibiting the importance of the first two dimensions of the multiple correspondence analysis

\begin{tabular}{llll}
\hline Dimension & Eigen value & Variance & Adj. variance \\
\hline 1 & 0.166 & 12.4 & 65.4 \\
2 & 0.138 & 10.4 & 28.8 \\
3 & 0.102 & 7.7 & 3.4 \\
4 & 0.095 & 7.2 & 1.4 \\
5 & 0.091 & 6.8 & 0.5 \\
\hline
\end{tabular}


disparity with $88 \%$ female respondents, corresponding to the large over-representation of women in nursing and healthcare professions, and might therefore reflect an oversampling of nurses and health workers. Nevertheless, our study utilises a convenience sampling approach and it is therefore not our intention to generalise to the broader population of health workers, but rather to point at possible trends in modes of bodily health investments, which then calls for further theorising and empirical research on the topic.

\section{Analysis}

We analyse response patterns among Danish health professionals and students on modes of body investment by conducting a multiple correspondence analysis on 12 variables divided into 28 variable categories.

Three supplemental demographic variables are included to group responses according to demographic characteristics. To avoid disproportioning the map, each category should contribute with at least the same contribution as the average contribution (of all categories) on at least one dimension (Roux and Rouanet 2010). A total of eight categories (medium food score, no active holidays, normal BMI, overweight BMI, no to new age activities, no stress, some stress, and feel stressed) do not exceed the average on at least one dimension, and the variable stress does not contain any categories with contributions above the cut-off. However, as Roux and Rouanet (2010, p. 71) note, distances between points over 0.5 in these maps can be considered notable, which is an argument for keeping all the categories in the analysis and at the same time urge caution when interpreting them. The first and second dimensions account for approximately $23 \%$ of the overall explained variance. The amount of explained variance levels off at the third dimension, meaning the only sensible dimensions to interpret are the first and second. The explained variance on the first dimension is underestimated because traditional MCA creates artificial additional dimensions (See Abdi and Valentin 2007 for a more detailed and technical explanation). Both Benzécri (1969) and Greenacre (1993) have suggested corrections for how to calculate the correct variance. The adjusted variance is about $65 \%$ for dimension 1 and almost $29 \%$ on the second dimension. Thus, we find considerable improvement on the key dimensions when adjusting the explained variance. In comparison, the third dimension drops by about four percentage points.

The variable categories are placed according to their respective contribution to the first and second dimensions as seen in Fig. 1:

In Fig. 2, we exhibit the cloud of categories. We interpret the structure of the bodily investment strategies as a two-dimensional space. The first dimension (horizontal) is interpreted as the distinction between an instrumental (left) and a natural-holistic (right) body investment strategy. The second dimension (vertical) is interpreted as a distinction between engagement (top) and absence (bottom). 

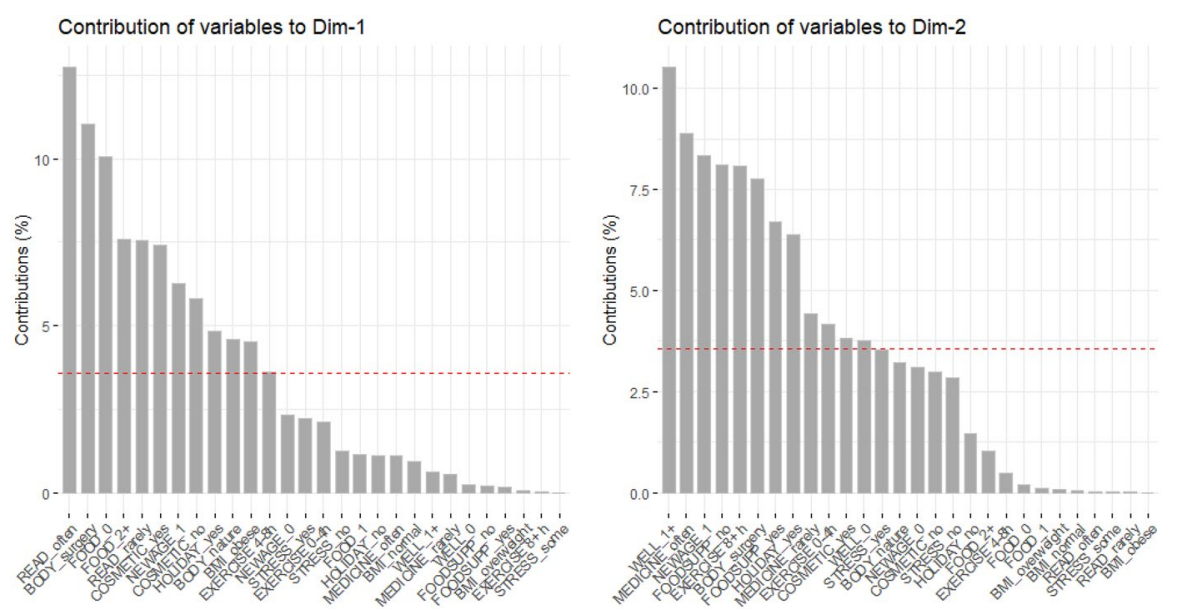

Fig. 1 Contribution to the overall variance by variable categories to the first and second dimension. The dotted line indicates the average contribution (3.7\%) given the number of categories

\section{The first dimension: interventionistic vs naturalistic body}

The first dimension is displayed on the horizontal axis of Fig. 2. The left side describes subjects with an instrumental and interventionistic relationship to their own bodies. These individuals are more open to cosmetic changes, feel more stressed, rarely read, do not eat organic food or vegetables, and frequently consume medicine. Challenges are met with hard, invasive, fast, and non-natural means. We also find the most overweight subjects in this space. On the right side of the map, the emphasis is more on soft, natural, and indirect investments, primarily through the diet and mental body. In general, we could say that investments among individuals placed in the left side are relatively dramatic and that benefits are expected in the short term, whereas investments of subjects positioned on the right side are soft and the benefits are expected in the long term. Extensive exercise ( $8 \mathrm{~h}$ per week or more) are found somewhere in the middle between the two, albeit this variable is substantially more present on the second dimension.

The right side of the map is interpreted as a group of individuals having a more holistic and naturalistic approach to their own bodies. These subjects oftentimes read, eat vegetables and organic food, exercise regularly, and engage in meditation or yoga. Radical body changes are thought of critically, and the body is understood as a natural thing that should not be corrected through taking medicine but instead through diet. Positive results of this body perception can be seen on the low scores on individual stress. As the map shows, the diet body is mainly active on the first dimension. The same applies to the surgical body, where negative coordinates signal openness to body alterations and vice versa. Medicine intake is also present on the first dimension, where negative coordinates indicate the frequent consumption of medicine. To a large extent, reading is also spread out along this dimension, which 


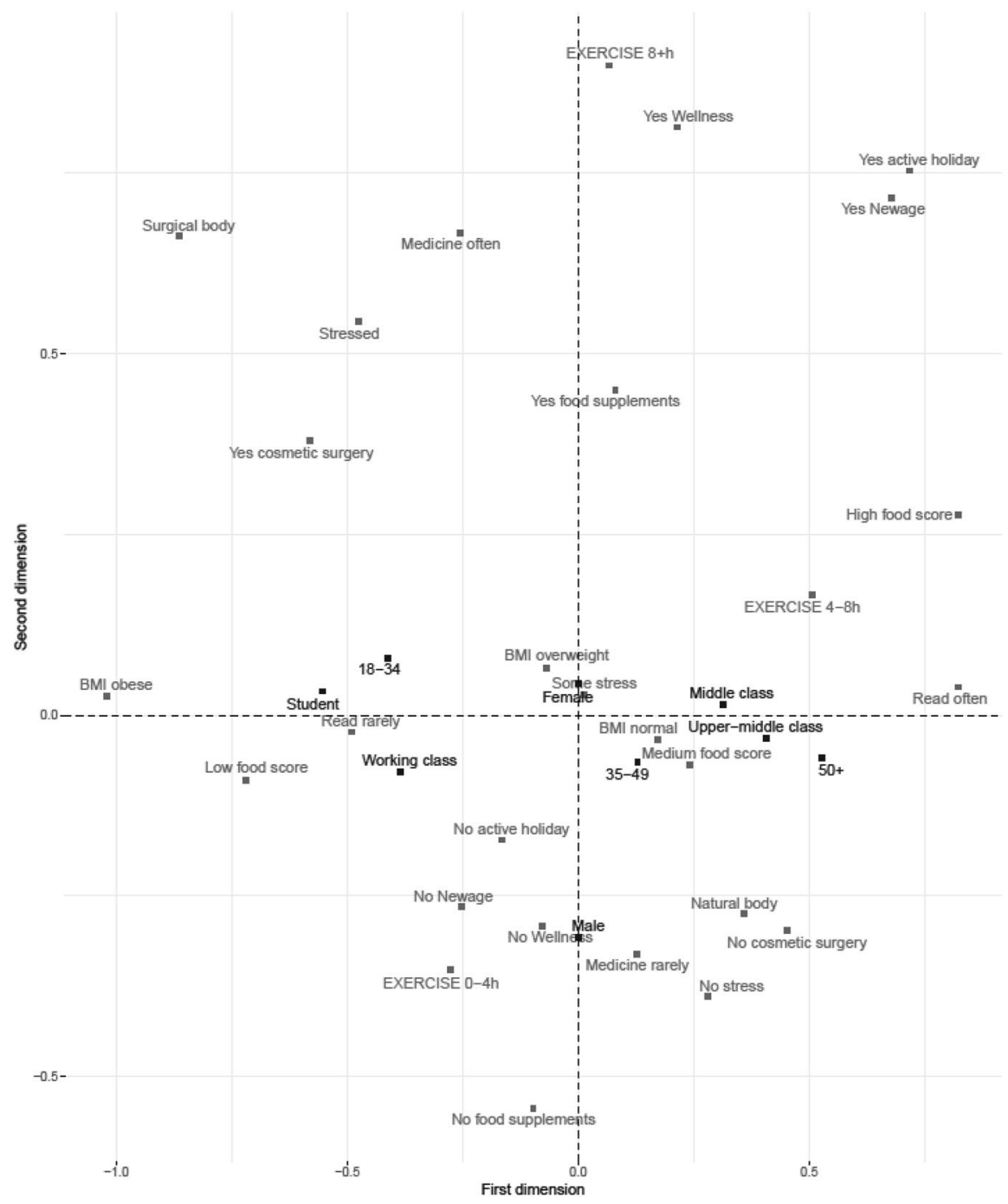

Fig. 2 The cloud of categories. A map placing indicators of body investment modes into distinct practices. The categories normal BMI and age group 35-49 are placed on top of each other. The same holds for the overweight BMI category

indicates that the classic conception of cultural capital is a strong indicator of this distinction. It is clear from Fig. 2 that the chemical and surgical body are in stark contrast to the diet body. The first body perception (left side) can be understood as a cultured body that should be continuously optimised and corrected-not only when illness, injuries, or accidents occur but also for aesthetic reasons. This is not the case for the natural body, where the natural appearance of the body is more accepted and is primarily maintained through diet. 


\section{The second dimension: engagement vs absence}

Interpreting the second dimension (vertical), we argue that the top half of the map is characterised by engagement, that is, by frequent physical exercise, engaging in new age activities, use of food supplements, and a preference for active holidays. In contrast, the bottom half of the map is characterised by passivity, moderation, and non-investments in terms of medicine intake, exercise, and food consumption. By the top pole, intense physical exercise ( $8 \mathrm{~h}$ or more), prioritising active holidays, and new age activities (e.g. yoga) are present. By the bottom pole, subjects placed here are characterised by not using food supplements, rarely performing physical exercise, rarely consuming medicine, not feeling stressed, and not engaging in new age activities. These can be considered polarisations between an active and investing body orientation and a more moderate and relaxed body orientation. In particular, this polarisation is related to new age and wellness aspects of the mental body. In the following, we present a more thorough analysis of the distinctions present in the four quadrants of the cloud of categories (see Fig. 3 below).

\section{Distinctions in bodily health investment strategies}

The quadrants display substantial polarisations in bodily activities related to the five types of bodily health investments of embodied health capital.

In the top-left quadrant, body perception can best be described as instrumental and interventionistic. These individuals are open to cosmetic changes and body surgery and more frequently consume medicine (to avoid or prevent sickness) than the other subjects. These individuals also feel the most stressed compared to individuals positioned in the three other quadrants. In summary, the physical body, chemical body, and surgical body dominate this quadrant and the subjects placed here seem to

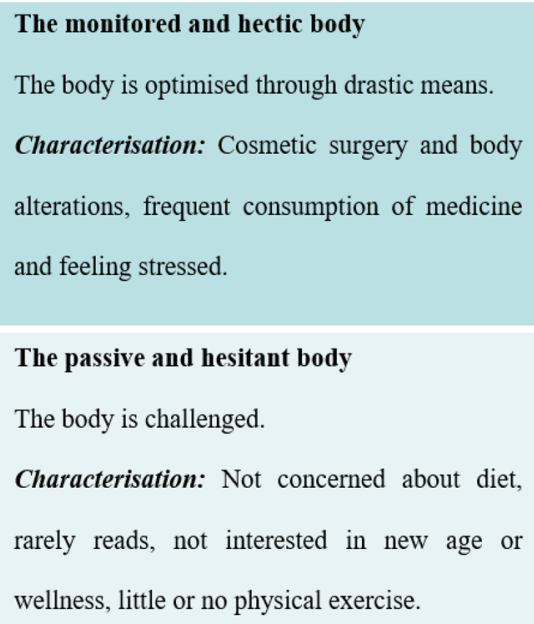

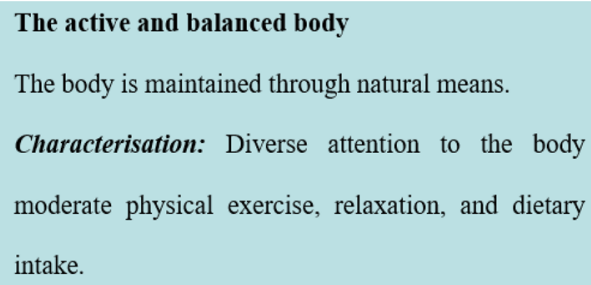

Fig. 3 A summary of the four distinct body investment strategies found in the cloud of categories 
emphasise optimisation as opposed to maintenance of the body, also through drastic means.

The bottom-left quadrant is composed of individuals who do not read frequently, or engage in any type of new age or wellness activity, which reveals a low stimulation of the mental body. They also rarely eat organic food or vegetables and do not use food supplements. The individuals present here also exercise the least, which is visible through their dislike of active holidays and a physical exercise rate of $0-4 \mathrm{~h}$ a week. This points to the body, in terms of physical and mental health, being challenged, while there is no mental effort to act. We characterise this as non-investment in the body.

The top-right quadrant displays attitudes toward more holistic practices. These individuals are characterised by their practice of wellness and new age activities, being physically active through frequent exercise and active holidays, eating healthy food, and taking food supplements, minerals, and vitamins. They are also the most frequent readers among the cases observed. In this quadrant, the mental body, the physical body, and the diet body are present. The body is balanced and regulated through a variety of physical exercise, relaxation, and dietary investments.

The bottom-right quadrant is characterised by individuals strongly opposed to altering the body with surgery or cosmetics. This is shown by their preference for the natural body. At the same time, these individuals distinguish themselves from the top-right quadrant by being less active in terms of physical investments. Instead, they display absence of investment by rarely consuming medicine. Interestingly, they also feel the least stressed among the subjects in this study. They seem to have a body that does not necessarily crave attention, which is seen in how they distance themselves from powerful/demanding investment strategies such as surgery or physical exercise. The body is maintained not through hectic interventions but mainly through the diet body.

\section{The cloud of individuals-variations across demographic characteristics}

To further distinguish the response patterns on modes of bodily health investments, we inspect Fig. 4 (i.e. the cloud of individuals). We group the sample into subgroups based on their social class status, age (in groups), and gender. The lack of any gender differences could be due to the large skewness in the sample, which should be corrected in future research. However, we find suggestive evidence of a distinction between the student- and working-class subjects and the middle- and upper-middle-class subjects (see top-left plot of 'Fig. 4') on the first dimension, while there appears to be only minor distinction on the second dimension (i.e. absence vs engagement). These groups are interpreted together because the confidence ellipses overlap. Hence, the middle class and upper middle class seem to be engaged with their body in a holistic and naturalistic manner, which entails a preference for the mental body and the diet body (as measured by the food index and not the question on supplements). Oppositely, the participants coded as student and working class seem to generally view the body in a passive and 

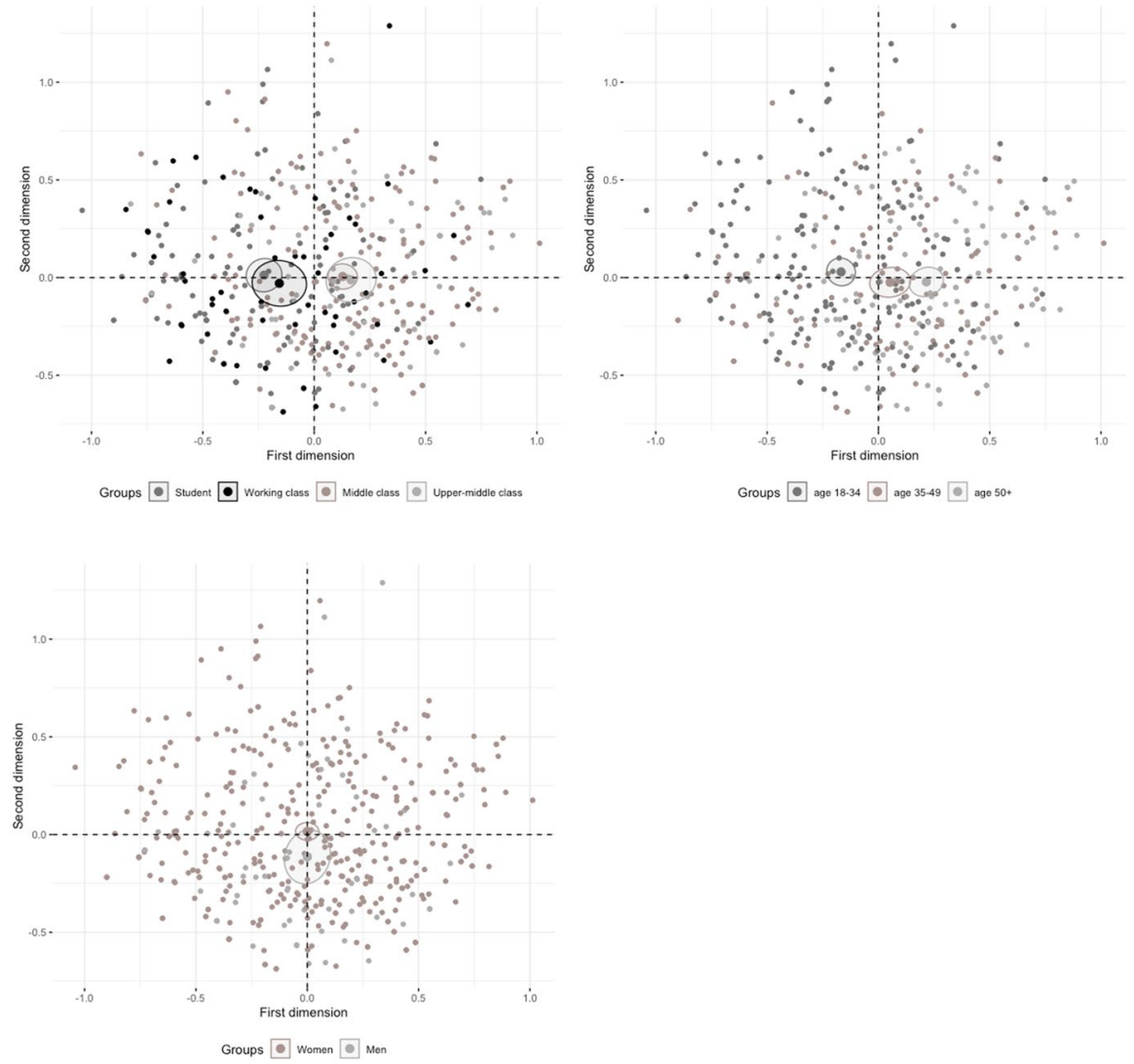

Fig. 4 Three plots representing the cloud of individuals with point estimates and associated $95 \%$ confidence ellipses of the supplemental variable categories

hesitant manner as defined in Fig. 3. To further test this, it could be fruitful to look more into how cultural and economic capital play into this contrast, as the higher income groups have more resources to maintain their body through investments through active holidays, wellness, yoga, meditation, etc. This could be the subject of a new research article utilising MCA on health capital investments. Furthermore, generational gaps and relation between age and class could also be the subject of further research assessed by the position and distance in the individual maps (see Fig. 3). It seems evident that the three age groups (see Fig. 4) follow the distribution of the four class differentiations.

These distinctions are further confirmed when inspecting the top-right plot. Examining the age groups, we find that the younger group more or less corresponds to the student category, while the older group resembles the naturalistic body view characteristic of the middle- and upper-middles class. Thus, on average, the latter group are more reluctant towards cosmetic interventions and medicine intake than the former and also feel less stressed on average. The older group 
of health professionals in this sample thus feel less stressed in their daily lives and seem to perceive the body as something natural that does not necessarily need to be activated through training, medicine, or surgical interventions. Oppositely, the young group seem to eat less healthy, feel more stressed, and rarely read literature. As previously mentioned, social class and age are primarily effectual on the first dimension, where they illuminate the spectrum of the instrumentalised and naturalised body characterised by dietary habits, body views, and stimulation of the mind.

By combining the findings from both clouds, we observe subjects in the top-left quadrant are split into two groups, characterised by different modes of bodily health investments. The distribution of individuals indicates that this cannot be explained by social class or age differences. We find a group of subjects (1) who intervene using fast, non-natural, and invasive tools and techniques (i.e. chemical and surgical body) and another (2) with a high BMI. The latter are situated closer to the passive and hesitant body characteristic of the bottom-left quadrant. Likewise, the active and balanced body (i.e. top-right quadrant) can be split into two distinct body investment strategies: (1) those who often read, exercise moderately, and have a healthy diet and (2) those who engage in new age and wellness activities and prefers an active holiday. The latter distinction explicates a differentiation of the mental and diet body. The variance of the two clouds substantiates these statements, as both clouds clearly reveal how the top of the map has a larger variance between the cases than the bottom of the map. This means that for negative coordinates on the second dimension, individuals demonstrate clear non-investment in which categories that indicate absence are very closely positioned. In contrast, participants placed in the top half of the map have a broader perspective on body investment, which illustrates their clear engagement to distinct activities from more instrumental and invasive to more holistic practices.

\section{Discussion}

Our main findings tap into four distinct subject areas within healthcare studies drawing on a Bourdieusian line of theory.

First, healthism and the construction of social space: Healthism is superseding national borders (Crawford 1980) and is embedded in broader transnational fields involving the UN (WHO) and EU (OECD 2009) (Bourdieu 1998, 2001). Dominant international positions structure fields, for instance workplace fields and the healthcare field (Larsen and Harsløf 2019) and constitute homologies across fields that are in a production-consumption relationship (Wang 2016). Rather than assigning an ontological status to social facts, we construct the scientific sociological object (Bourdieu et al. 1991, pp. 32-35) as part of an overall social space focusing on Danish healthcare professions. This social space has similarities to a healthcare field (Collyer et al. 2017) in which symbolic, cultural, or scientific capitals are central objects of struggle. However, body performances, eating, or training preferences of professions are peripheral resources in the field, yet obviously related to the overall structure of the healthcare field. We have shown that differentiation in the space is 
related to social positions, but also to age, which could indicate that this is a generational issue as much as an issue of class domination. We have revealed how relations of domination in the healthcare field are also related to different and opposed investment strategies on the body (instrumental vs. naturalistic practice, engagement vs. absence, hectic vs. balanced). Different and opposed body practices are present in this empirical data, and while health initiatives promote health, they also lead 'low active' employees to feel incompetent and exclude themselves, thus supporting social inequality (Rossing 2017).

Second health capital and related concepts: Similar to health capital, cultural health capital (Shim 2010), personal capital (Scambler and Newton 2011), healthcare capital (Harley et al. 2011), and healthcare choice (Collyer et al. 2015) concepts, all integrate Bourdieu's dyad of habitus, capital, and field, and they offer substantial insight into the complex relations of dominance and struggles about practices and values in different healthcare fields and institutions. Also, like health capital, these concepts construct objects as 'relational', as opposed to theories of competence, profession, patients, or organisations. The main difference is that health capital sets out to understand how social investment practices in everyday life, acquiring its social and symbolic energy from health and well-being, shape different and opposed body investments among health care professions. We can also ask whether we did study a specific kind of cultural capital, physical capital, or body capital (Bourdieu 1986)? We argue that by constructing health capital and its subcomponents, we added concepts to understanding social practices and strategies that former capitals did not accomplish. Transformed social space and fields and the fitness, yoga, meditation, and surgery industries constitute a background for health capital as a new possible resource that can be applied as a social distinction.

Third, dominant and dominated health capital: Dominant social groups will act and look 'natural' when investing in the body, since their values are naturalised, similar to how the upper middle class dominates the school and educational system (Bourdieu and Passeron 1977), whereas some dominated social groups, according to the Bourgeois model (Skeggs 2011, p. 509), 'overinvest' in the body (e.g. fitness, diet, and surgery). The dominant social groups perceive this as vulgar, 'not appropriate', or as bizarre. As an example, oversized physiognomy (muscles) will only fit in specific social fields and positions in that field, whereas the inner body and investment in the mental body may be valued among the cultural elite. Nevertheless, capital is nothing in itself it is the position in a social space or field that includes or excludes practices. Capital can, when invested appropriately, be converted. In this sense, economic, cultural, social, and health capital can be transformed into each other; for example, health capital can be converted to economic capital (a new job), which, in turn, can be converted into cultural capital (ability to afford an education) and social capital (access to networks).

Fourth, capital differentiation: In the four quadrants of the map, we could point to four general investment strategies that-especially in the top half of the mapcan be further broken down to more particular body investment strategies (see 'Fig. 4'). If the correspondence analysis has sparked any important considerations, it would be to re-evaluate the composition of investment in the mental body. Maybe it would be more reasonable to divide it into a mental body category directed towards 
engagement with yoga, new age activities, and mindfulness, and a cultural body that relates more to cultural consumption such as reading, museum and theatre visits, and taste in art.

As for future research, we hypothesise that examinations of relations between professions and patients will diverge even more. Patients' habitus via the amount and composition of capitals is extremely diverse and heterogeneous, and direct observations could display how 'fit, slim, and healthy' professions handle 'unfit, fat, and unhealthy' patients. How is the direct body-to-body interaction articulated in a field that promotes objective knowledge, evidence, standards, and biomedical thinking? Unfortunately, we expect that similarities in habitus constitute better treatment, as in the case of rehabilitation (Guldager et al. 2018).

Anyway, health capital offers a research programme applicable across a broad range of empirical areas and consolidates existing capital-related concepts in health sociology. This study provides a pioneering combination of Bourdieu's relational sociology and suggests a typology of five ways of investing in the body. Others have studied single investments in surgery, physical training, or diet (e.g. Morgan 1991; Markula-Denison and Pringle 2007; Turner 2003), but they are integrated and combined in health capital.

\section{Conclusion}

The aim of this article has been to explore and characterise how professionals and students in the health sector use different strategies or modes when investing in their bodies. To answer this, we have used the theoretical concept of health capital developed by (Larsen et al. 2013). A multiple correspondence analysis suggests that some groups within the Danish healthcare field have a more instrumental body perception — such as the young age group — while the upper-middle-class and older respondents have a more holistic and naturalistic perception of the body. The cloud of categories shows patterns of preference primarily distinguished on the first dimension (about $65 \%$ of explained variance), while the second dimension is more about the frequency of investment (about 29\% of explained variance). The physical body is primarily distinguished on the latter, while the diet and surgical body are primarily active on the first dimension. The chemical body is both visible on the first and second dimension, where positive coordinates on the second dimension signal frequent medicine use and vice versa. The mental body is primarily active on the second dimension, where positive coordinates indicate engagement with new age and wellness activities, although it is also effectual on the first dimension, where positive coordinates signal frequent reading. In conclusion, it appears feasible to think of body practices related to health in terms of the five types of body investment of embodied health capital.

Acknowledgements Thanks to Margaretha Jarvinen and the inspiration of questionnaire used in an alcohol survey. Drinking to the limit: Alcohol, social status, and health governance (2014). 
Open Access This article is licensed under a Creative Commons Attribution 4.0 International License, which permits use, sharing, adaptation, distribution and reproduction in any medium or format, as long as you give appropriate credit to the original author(s) and the source, provide a link to the Creative Commons licence, and indicate if changes were made. The images or other third party material in this article are included in the article's Creative Commons licence, unless indicated otherwise in a credit line to the material. If material is not included in the article's Creative Commons licence and your intended use is not permitted by statutory regulation or exceeds the permitted use, you will need to obtain permission directly from the copyright holder. To view a copy of this licence, visit http://creativecommons.org/licen ses/by/4.0/.

\section{References}

Abdi, H., and D. Valentin. 2007. Multiple factor analysis (MFA). In Encyclopedia of measurement and statistics, ed. N. Salkind, 1-14. Thousand Oaks: Sage.

Bachelard, G. 1968. The philosophy of no: a philosophy of the new scientific mind, translation by G.C. Waterston. (La philosophie du non). New York: Orion Press.

Benzécri, J.P. 1969. Statistical analysis as a tool to make patterns emerge from data. In Methodologies of pattern recognition, ed. Watanabe, S. New York: Academic Press. Dekker: Correspondence Analysis Handbook, 35-74.

Bertilsson, T.M. 2004. The elementary forms of pragmatism: On different types of abduction. The European Journal of Social Theory 7 (3): 371-389.

Bourdieu, P. 1977. Outline of a theory of practice. Cambridge: Cambridge University Press.

Bourdieu, P. 1981. Ce que parler veux dire: L'économie des échanges linguistiques [That talk mean: The economics of linguistic exchanges]. Paris: Fayard.

Bourdieu, P. 1984 [1979]. Distinction: A social critique of the judgement of taste (translated by Richard Nice). Cambridge: Harvard University Press.

Bourdieu, P. 1985. Le champ religieux dans le champ de manipulation symbolique [The religious field in the symbolic manipulation of field]. In Les nouveaux clercs-Prêtres, pasteurs et spécialistes des relations humaines et de la santé [The new clergy-priests, pastors and specialists in human relations and health], ed. G. Vincent, 255-261. Labor et Fides: Geneves.

Bourdieu, P. 1986. The forms of capital. In Handbook of theory and research for the sociology of education, ed. J.G. Richardson. New York: Greenwood Press.

Bourdieu, P. 1998. Acts of resistance: Against the new myths of our time. London: Polity Press.

Bourdieu, P. 1999. The weight of the world: Social suffering in contemporary society. London: Polity Press.

Bourdieu, P. 2001. Firing back against the tyranny of the Market 2. New York: The New Press.

Bourdieu, P., Chamboredon, J.C., Passeron, J.C. and Krais, B. 1991. The craft of sociology. DE GRUYTER.

Bourdieu, P., and S. Farage. 1994. Rethinking the state: Genesis and structure of the bureaucratic field. Sociological Theory 12: 1-18.

Bourdieu, P., and J.C. Passeron. 1977. Reproduction in education, society and culture. London: Sage Publications.

Brewis, A.A., A. Wutich, A. Falletta-Cowden, and I. Rodriguez-Soto. 2011. Body norms and fat stigma in global perspective. Current Anthropology 52 (2): 269-276.

Canguilhem, Georges. 1988. ldeology and rationality in the history of the life sciences. Cambridge: The MIT Press.

Carleheden, M. 2016. What conception of the theoretical does 'theorizing' presuppose? Comment on Richard Swedberg's 'Before theory comes theorizing or how to make social science more interesting.' British Journal of Sociology 67: 36-42.

Collyer, F.M., K.F. Willis, M. Franklin, K. Harley, and S.D. Short. 2015. Healthcare choice: Bourdieu's capital, habitus and field. Current Sociology Monograph 63 (5): 685-699.

Collyer, F.M., K.F. Willis, and S. Lewis. 2017. Gatekeepers in the healthcare sector: Knowledge and Bourdieu's concept of field. Social Science and Medicine 186: 96-103.

Crawford, R. 1980. Healthism and the medicalization of everyday life. International Journal of Health Services 10 (3): 365-388. 
Di Franco, G. 201. Multiple correspondence analysis: one only or several techniques? Quality \& Quantity 50 (3): 1299-1315.

Greenacre, M. 1993. Correspondence analysis in practice. London: Academic Press.

Guldager, R., I. Poulsen, I. Egerod, L.L. Mathiesen, and K. Larsen. 2018. Rehabilitation capital: A new form of capital to understand rehabilitation in a Nordic welfare state. Health Sociology Review 27 (2): 199-221.

Harley, K., K. Willis, S. Short, F. Collyer, J. Gabe, and M. Calnan. 2011. Navigating public/private healthcare boundaries: Choice and healthcare capital, Proceedings of 2011 TASA Conference: Local Lives/Global Networks, 28 Nov-1 Dec, Newcastle, Australia EJ ISBN 9780646567792 [Refereed Conference Paper].

Järvinen, M., C.H. Ellersgaard, and A.G. Larsen. 2014. Drinking to the limit: Alcohol, social status and health governance. Drugs - Education Prevention and Policy 21 (4): 283-298.

Larsen, K. 2009. Kroppe: sundhed og social ulighed. In: Glasdam S, red. Folkesundhed - i et kritisk perspektiv. 1. udg. Kbh.: Nyt Nordisk Forlag; 2009. s. 188-218. (Body, Health and social Inequality).

Larsen, K., and V. Morrow. 2009. Social position and young people's health: A Bourdieu'ian critique of dominant conceptualisations of social capital. Praktiske Grunde: Nordisk tidsskrift for kultur-og samfundsvidenskab 3: 7-30. http://praktiskegrunde.dk/2009/praktiskegrunde(2009-3d)larsen-morro w.pdf.

Larsen, K., and G.I. Hansen. 2014. Social ulighed i sundhed - mere vilkår end valg: Indsigter og forklaring på norske forhold. Nasjonal kunnskapsinnsamling om sosial ulikhet og helse. Oslo: HiOA. Dahl, E., Bergsli, H. \& van der Wel, K. A. (red.). Høgskolen i Oslo og Akershus, s. 1-69 69 s. (Social Inequality in Health, more condition than choice).

Larsen, K., and I. Harsløf. 2019. Promoting health as a form of capital: The transformation of the Danish healthcare field as experienced by private healthcare professionals. In Navigating private and public healthcare experiences of patients, doctors and policy-makers, 1st edn, ed. F. Collyer, and K. Willis, 201-223. Singapore: Palgrave Macmillan.

Larsen, K., Cutchin, M., and I. Harsløf. 2013. Health capital: New health risks and personal investments in the body in the context of changing nordic welfare states. I I. Harsløf, \& R. Ulmestig (red.), Changing social risks and social policy responses in the nordic welfare states, s. 165-188. New York: Palgrave Macmillan.

Le Roux, B., and H. Rouanet. 2010. Quantitative applications in the social sciences: Multiple correspondence analysis. Thousand Oaks: SAGE Publications Ltd.

Lupton, D. 2016. The quantified self: A sociology of self-tracking. Cambridge: Polity Press.

Markula-Denison, P., and R. Pringle. 2007. Foucault, sport and exercise: Power, knowledge and transforming the self. London: Routledge.

Morgan, K.P. 1991. Women and the knife: Cosmetic surgery and the colonization of women's bodies. Hypatia 6 (3): 25-53.

OECD. 2009. Sickness, disability and work: Breaking the barriers. Paris: OECD.

Rose, N. 2003. Neurochemical selves. Society 41: 46-59. https://doi.org/10.1007/BF02688204.

Rose, N. 2007. The politics of life itself. Biomedicine, power, and subjectivity in the twenty-first century. Princeton: Princeton University Press.

Rossing, H. 2017. Role conflict, facework and vulnerable identity. Doctoral study at the Norwegian School of Sport Sciences.

Scambler, S., and P. Newton. 2011. Capital transactions, distruptions and the emergence of personal capital in a lifeworld under attack. Social Theory \& Health 9 (2): 130-146.

Shim, J.K. 2010. Cultural health capital: A theoretical approach to understanding health care interactions and the dynamics of unequal treatment. Journal of Health and Social Behavior 51 (1): 1-15.

Skeggs, B. 2011. Imagining personhood differently: Person value and autonomist working-class value practices. The Sociological Review 59 (3): 496-509.

Skjøtt-Larsen, J. 2012. Cultural and moral class boundaries in a nordic context. European Societies 14 (5): 660-683. https://doi.org/10.1080/14616696.2012.720271.

Turner, B.S. 2003. McDonaldization linearity and liquidity in consumer cultures. Cambridge: Sage Journals University of Cambridge.

Tyler, I. 2015. Classificatory struggles: Class, culture and inequality in neoliberal times. The Sociological Review 63 (2): 493-511.

Wang, Y. 2016. Homology and isomorphism: Bourdieu in conversation with New Institutionalism. The British Journal of Sociology 67 (2): 348-370. https://doi.org/10.1111/1468-4446.12197. 
Publisher's Note Springer Nature remains neutral with regard to jurisdictional claims in published maps and institutional affiliations.

Kristian Larsen has been a professor at OsloMet University and Aalborg University since 2010 in Health, Sociology, and Education. He has published 141 articles including book. He has supervised $16 \mathrm{Ph} . \mathrm{D}$. students and he is the founder of the Nordic Bourdieu Organisation HEXIS: https://urldefense.proof point.com/v2/url? $\mathrm{u}=$ http-3A_www.hexis.dk\&d=DwIGaQ\&c=vh6FgFnduejNhPPD0fl_yRaSfZy8CW bWnIf4XJhSqx8\&r=pJkJ7F_3vDot2EBIJTbn5-MYrVqdgD9kmitFKREy_Jp1J8OB1dr0hoW1j--QhpHB $\& m=c m B 4 z r-A h 5 W t p S p Y c a c x x v F s c a S p d F c Y 4 z M y l c g L L h g \& s=b e u 3 u p x m-91 I 5 w G P 5 z G 5 D S H V$ vsYgDdu4rEW7jwaKc88\&e $=$ Leader of the Danish-Norwegian research collaboration https://urlde fense.proofpoint.com/v2/url? $\mathrm{u}=\mathrm{http}-3 \mathrm{~A} \_$_www.phlegethon. net\&d=DwIGaQ\&c=vh6FgFnduejNhPP D0f1_yRaSfZy8CWbWnIf4XJhSqx8\&r=pJkJ7F_3vDot2EB1JTbn5-MYrVqdgD9kmitFKREy_Jp1J8 OB1dr0hoW1j--QhpHB\&m=cmB4zr-Ah5WtpSpYcacxxvFscaSpdFcY4zMylcgLLhg\&s=VucL4grqKU t-ZAdWB3w4p7NDlebdjtmE8vawdasyTXE\&e= involving Ed. this book: https://www.palgrave.com/gp/ book/9789811373459.

Anette Lykke Hindhede has published 80 articles and bookchapters in a broad spectrum in Health Sociology and Educational sociology https://vbn.aau.dk/da/publications/?search=anette+lykke +hindhede\&originalSearch=anette+lykke+hindhede\&pageSize=50\&ordering=rating\&descending $=$ true $\&$ showAdvanced $=$ false $\&$ allConcepts $=$ true \&inferConcepts=true $\&$ searchBy $=$ PartOfNameOrTitle

Mikkel Haderup Larsen is a sociologist (Cand. Scient. Soc. Spring 2020) from the University of Copenhagen, Denmark. He is now a Ph.D. student at the University of Copenhagen and Roskilde

Mathias Holst Nicolaisen is a sociologist (Cand. Scient. Soc. Spring 2020) from the University of Copenhagen, Denmark. He is now a Ph.D. student at the University of Copenhagen and Roskilde

Frederik Møller Henriksen is a sociologist (Cand. Scient. Soc. Spring 2020) from the University of Copenhagen, Denmark. He is a research Assistant at Copenhagen University.

\section{Affiliations}

\section{Kristian Larsen 1,2,3 (1) Anette Lykke Hindhede ${ }^{4}$ Mikkel Haderup Larsen ${ }^{4,5}$. Mathias Holst Nicolaisen ${ }^{4,6}$. Frederik Møller Henriksen ${ }^{7}$}

Anette Lykke Hindhede

anlh@hum.aau.dk

Mikkel Haderup Larsen

mhl@soc.ku.dk

Mathias Holst Nicolaisen

mathiasn@ruc.dk

Frederik Møller Henriksen

fmh@jur.ku.dk

1 The University Hospitals Centre for Health Research (UCSF), University of Copenhagen,

Copenhagen, Denmark

2 RigsHospitalet, Ryesgade 27, 2200 Copenhagen, Denmark

3 Oslo Metropolitan University, Postbox 4, St. Olavs Plass, 0120 Oslo, Norway

4 Department of Culture \& Learning, Aalborg University, A. C. Meyers Vænge 15, 2450 Copenhagen, Denmark 
5 Department of Sociology, Copenhagen University, Øster Farimagsgade 5, 1353 Copenhagen K, Denmark

6 Department of Social Sciences and Business, Roskilde University, Universitetsvej 1, 4000 Roskilde, Denmark

7 Faculty of Law, Copenhagen University, iCourts, Karen Blixens Plads 16, 2300 Copenhagen S, Denmark 